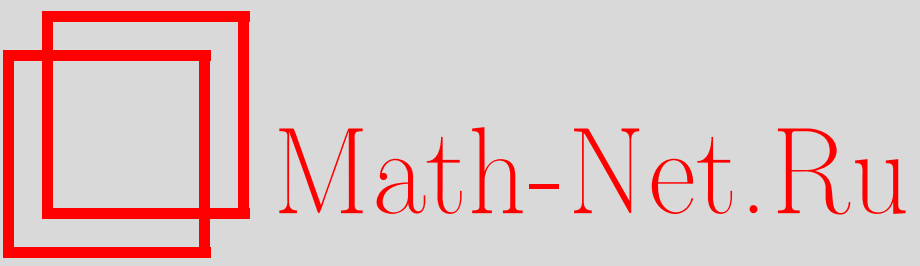

А. В. Забродин, Бездисперсионный предел уравнений Хироты в некоторых задачах комплексного анализа, ТМФ, 2001, том 129, номер 2, 239-257

DOI: https://doi.org/10.4213/tmf534

Использование Общероссийского математического портала Math-Net.Ru подразумевает, что вы прочитали и согласны с пользовательским соглашением

http://www.mathnet.ru/rus/agreement

Параметры загрузки:

IP: 54.198 .55 .26

26 апреля 2023 г., 10:32:11 
ТЕОРЕТИЧЕСКАЯ

И МАТЕМАТИЧЕСКАЯ

ФИЗИКА

Том 129, № 2

ноябрь, 2001

(C) 2001 г.

А.В. Забродин*

\section{БЕЗДИСПЕРСИОННЫЙ ПРЕДЕЛ УРАВНЕНИЙ ХИРОТЫ В НЕКОТОРЫХ ЗАДАЧАХ КОМПЛЕКСНОГО АНАЛИЗА}

Обсуждается интегрируемая структура, недавно обнаруженная в некоторых классических задачах теории функций одного комплексного переменного. Для односвязной области с аналитической границей в плоскости комплексного переменного рассматриваются задача конформного отображения, граничная задача Дирихле и двумерная обратная задача теории потенциала. На пространстве таких областей построено некоторое замечательное семейство вещественнозначных функционалов. Если рассматривать их как функции бесконечного множества переменных, которые являются подходящим образом определенными моментами области, любой функционал из этого семейства дает формальное решение перечисленных выше задач. Показано, что определенные таким образом функции удовлетворяют бесконечному множеству бездисперсионных уравнений Хироты. Это означает, что они являются $\tau$-функциями некоторой интегрируемой иерархии. Эта иерархия отождествляется с бездисперсионным пределом двумеризованной цепочки Тоды. В дополнение к нашим предшествующим результатам показано, что при более общем определении моментов указанная взаимосвязь не является специфичной для какого-нибудь одного решения уравнений Хироты, а отражает структуру самой иерархии.

\section{1. ВВЕДЕНИЕ}

Недавно было показано [1]-[3], что некоторые задачи комплексного анализа, такие как задача о конформном отображении, граничная задача Дирихле и двумерная обратная задача теории потенциала, обладают скрытой интегрируемой структурой. Оказывается, существует бесконечная иерархия нелинейных интегрируемых уравнений такая, что вариации искомых решений перечисленных выше задач при деформации данных задачи могут быть найдены путем решения уравнений иерархии. Иерархия, о которой идет речь, - это определенный скейлинговый предел иерархии уравнений двумеризованной цепочки Тоды, который обычно называется бездисперсионным пределом.

Задача о конформном отображении состоит в том, чтобы найти конформное отображение данной односвязной области в комплексной плоскости на единичный диск. Как следует из теоремы Римана, такое отображение существует и после наложения некоторых простых условий нормировки единственно (см., например, [4]). Более точно, пусть

${ }^{*}$ Институт биохимической физики РАН, Институт теоретической и экспериментальной физики, Москва, Россия 
$\gamma$ - замкнутая аналитическая кривая в комплексной плоскости, а $D_{+}, D_{-}-$соответственно внутренняя и внешняя по отношению к кривой области. Через $w(z)$ обозначим конформное отображение области $D_{-}$на внешность единичного круга, нормированное таким образом, что оно переводит $\infty$ в $\infty$, и его производная на бесконечности - вешественное положительное число.

Граничная задача Дирихле в области $D_{-}$ставится следующим образом: для данной функции $\psi(z)$ на кривой $\gamma$ надо найти гармоническую функцию $f(z, \bar{z})$ в $D_{-}$такую, что $f(z, \bar{z})=\psi(z)$ при $z \in \gamma$. Решение дается формулой

$$
f(z, \bar{z})=-\frac{1}{\pi i} \oint_{\gamma} \psi(\zeta) \partial_{\zeta} G(z, \zeta) d \zeta
$$

где $G(z, \zeta)$ - функция Грина задачи Дирихле в области $D_{-}$. Функция Грина однозначно характеризуется следуюшими свойствами [5]: а) она регулярна на бесконечности и гармонична везде в $D_{-}$, кроме точки $z=\zeta$, где она имеет логарифмическую сингулярность, $G(z, \zeta)=\ln |z-\zeta|+O(1)$ при $z \rightarrow \zeta$; б) она равна нулю на границе $\partial D_{-}=\gamma$, т.е. $G(z, \zeta)=0$ для всех $\zeta$ при $z \in \gamma$.

Для того чтобы уточнить, что мы понимаем под обратной задачей теории потенциала, допустим (в тех же предположениях, что и раньше), что область $D_{+}$заполнена фоновым электрическим зарядом с равномерной плотностью. Как доказывается в теории потенциала, потенциал $\Phi(z, \bar{z})$, создаваемый этим зарядом, непрерывен на границе вместе со своими первыми производными (компонентами электрического поля), т.е. $\Phi^{+}=\Phi^{-}, \partial_{z} \Phi^{+}=\partial_{z} \Phi^{-}, z \in \gamma$. Здесь $\Phi^{ \pm}-$это функции $\Phi$, ограниченные соответственно на областях $D_{ \pm} ; \Phi^{-}$гармонична везде в $D_{-}$, кроме бесконечности, где она имеет логарифмическую особенность, в то время как $\Phi^{+}$гармонична с точностью до члена, пропорционального $|z|^{2}$. Следовательно, функции $\Phi^{ \pm}$можно представить в виде рядов Тейлора (мультипольных разложений) в окрестностях нуля и бесконечности, соответственно. Без потери обшности предположим, что точка 0 лежит в $D_{+}$. Коэффициенты мультипольных разложений $\Phi^{+}$и $\Phi^{-}$являются гармоническими моментами $D_{-}$и $D_{+}$, соответственно. Обратная задача теории потенциала заключается в восстановлении формы области на основе одного из этих разложений, $\Phi^{+}$или $\Phi^{-}$. Для определенности предположим, что даны коэффициенты $\Phi^{+}$, и мы будем искать коэффициенты

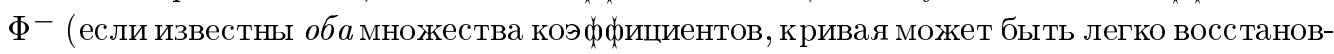
лена). Обзор обратных задач теории потенциала дан, например, в работе [6] (см. также приведенную там литературу).

Три указанных выше задачи тесно связаны друг с другом. Пусть, например, дано конформное отображение $w(z)$, тогда функция Грина может быть найдена явно с помощью формулы

$$
G(z, \zeta)=\ln \left|\frac{w(z)-w(\zeta)}{w(z) \overline{w(\zeta)}-1}\right|
$$

где черта означает комплексное сопряжение. В свою очередь, голоморфная по $z$ часть функции Грина является конформным отображением, хотя и по-другому нормированным. Чтобы получить отображение $w(z)$, нужно взять голоморфную часть функции 
$G(z, \infty)$. Что касается обратной задачи теории потенциала, отметим, что именно функция Грина осушествляет связь между $\Phi^{+}$и $\Phi^{-}$. В самом деле, введем модифицированный потенциал $\widetilde{\Phi}$, которьй есть $\Phi$ за вычетом логарифмической особенности на бесконечности, так что функция $\widetilde{\Phi}^{-}$гармонична в области $D_{-}$, включая $\infty$. Тогда в соответствии с формулой (1) можно записать

$$
\widetilde{\Phi}^{-}(z, \bar{z})=-\frac{1}{\pi i} \oint_{\gamma} \widetilde{\Phi}^{+}(\zeta, \bar{\zeta}) \partial_{\zeta} G(z, \zeta) d \zeta
$$

Напомним основные результаты работ [1]-[3]. Обозначим через $t_{k}, k \geqslant 1$, гармонические моменты области $D_{-}$, а через $t_{0}$ площадь области $D_{+}$(деленную на $\pi$ ):

$$
t_{k}=-\frac{1}{\pi k} \int_{D_{-}} z^{-k} d^{2} z, \quad t_{0}=\frac{1}{\pi} \int_{D_{+}} d^{2} z
$$

Заметим, что $t_{k}$ - вообще говоря, комплексные числа, а $t_{0}$ вещественно. В нижеследующих формулах комплексно-сопряженные моменты $\bar{t}_{k}$ считаются независимыми переменными. Введем операторы

$$
D(z)=\sum_{k \geqslant 1} \frac{z^{-k}}{k} \partial_{t_{k}}, \quad \bar{D}(\bar{z})=\sum_{k \geqslant 1} \frac{\bar{z}^{-k}}{k} \partial_{\bar{t}_{k}}
$$

и

$$
\mathcal{D}(z, \bar{z})=\partial_{t_{0}}+D(z)+\bar{D}(\bar{z}) .
$$

Существует вешественнозначная функция $F$ переменных $t_{0},\left\{t_{k}\right\},\left\{\bar{t}_{k}\right\}$ такая, что конформное отображение $w(z)$ дается формулой

$$
w(z)=z e^{-\frac{1}{2} \partial_{t_{0}}^{2} F-\partial_{t_{0}} D(z) F}
$$

Эта функция имеет явное интегральное представление. Далее, функция Грина граничной задачи Дирихле представляется в виде

$$
G\left(z_{1}, z_{2}\right)=\ln \left|z_{1}^{-1}-z_{2}^{-1}\right|+\frac{1}{2} \mathcal{D}\left(z_{1}, \bar{z}_{1}\right) \mathcal{D}\left(z_{2}, \bar{z}_{2}\right) F
$$

Отметим, что гипотеза о существовании формул такого типа была впервые высказана Тахтаджяном, строгое доказательство и обсуждение можно найти в работе [7].

Функция $F$ является бездисперсионным пределом логарифма $\tau$-функции иерархии двумеризованной цепочки Тоды. Она удовлетворяет бездисперсионным уравнениям Хироты

$$
\begin{gathered}
\left(z_{1}-z_{2}\right) e^{D\left(z_{1}\right) D\left(z_{2}\right) F}=z_{1} e^{-\partial_{t_{0}} D\left(z_{1}\right) F}-z_{2} e^{-\partial_{t_{0}} D\left(z_{2}\right) F} \\
z_{1} \bar{z}_{2}\left(1-e^{-D\left(z_{1}\right) \bar{D}\left(\bar{z}_{2}\right) F}\right)=e^{\partial_{t_{0}}\left(\partial_{t_{0}}+D\left(z_{1}\right)+\bar{D}\left(\bar{z}_{2}\right)\right) F}
\end{gathered}
$$

Бездисперсионная иерархия Тоды является примером универсальной иерархии уравнений Уизема, введенной в работах [8]. Последняя представляет собой многомерное 
обобщение иерархий уравнений гидродинамического типа [9], [10]. Известно, что уравнения иерархии Тоды имеют бесконечное множество решений. Решения могут быть параметризованы [11] каноническими отображениями в двумерном фазовом пространстве таким образом, что каждое решение соответствует канонической паре функций (в работе [11] они были названы твисторными данными решения). В действительности это эквивалентно характеризации решений с помошью струнных уравнений.

В рамках подхода, развитого в работе [2], конформные отображения описывались одним специальным решением бездисперсионной двумеризованной иерархии Тоды. Роль других решений, если они вообше имеют отношение к конформным отображениям, оставалась неясной. В этой работе мы как раз занимаемся этим вопросом. Обобщая предыдушие результаты, мы показываем, что этот же класс конформных отображений может быть описан любым решением иерархии.

Это описание достигается с помошью другого, более обшего определения моментов области. Как и раньше, моменты являются независимыми потоками иерархии. Однако меняется их связь с формой области, и для их определения нужны некоторые дополнительные данные. Они вводятся заданием вешественно-аналитической функции на комплексной плоскости, которую мы обозначим $\sigma(z, \bar{z})$. В контексте обратной задачи теории потенциала эта функция играет роль плотности фонового заряда, которая была постоянной в стандартной формулировке задачи. Более точно, мы предположим, что функция $\sigma(z, \bar{z})$ задана на всей комплексной плоскости и зафиксирована раз и навсегда, тогда как форма области может меняться. Фоновый заряд имеет плотность $\sigma(z, \bar{z})$ внутри области и 0 вне ее. Обратная задача потенциала ставится так же, как и раньше. Моменты, однако, определяются теперь по отношению к плотности $\sigma$. В дальнейшем мы будем называть эту задачу обобщенной обратной задачей теории потенциала.

Этот результат свидетельствует о том, что именно иерархия уравнений Хироты (а не какое-либо специальное струнное уравнение или другие соотношения, зависящие от выбора решения) отражает интегрируемую структуру конформных отображений и задачи Дирихле.

В разделе 2 мы вводим в рассмотрение функцию $F$, которая определяется с помощью некоторого вариационного принципа, и находим ее первые производные. Они дают формальное решение обобшенной обратной задачи теории потенциала. Формальные решения задачи о конформном отображении и задачи Дирихле даются вторыми производными функции $F$. На вторые производные этой функции имеется бесконечный набор соотношений, которые можно объединить в (бездисперсионньй предел) уравнения Хироты. Эти вопросы обсуждаются в разделе 3. Наконец, раздел 4 посвящен альтернативному подходу к бездисперсионным интегрируемым иерархиям, который позволяет вывести уравнения Лакса-Сато для конформных отображений.

\section{2. ОБОБЩЕННАЯ ОБРАТНАЯ ЗАДАЧА ТЕОРИИ ПОТЕНЦИАЛА}

Обратная задача теории потенциала - возможно, самый удобный контекст для определения $\tau$-функции кривых. Следуя идее работы [3], мы введем $\tau$-функцию с помощью электростатического вариационного принципа. Исходные данные и обозначения такие же, как в разделе 1. 
Напомним, что область $D_{+}$заполнена фоновым электрическим зарядом. Будем рассматривать распределения заряда, не обязательно равномерные, характеризуюшиеся вешественно-аналитической функцией $\sigma(z, \bar{z})$. Примем, что эта функция задана во всей комплексной плоскости, причем плотность фонового заряда равна $-\sigma$ внутри $D_{+}$и 0 вне $D_{+}$. Если функция $\sigma$ фиксирована, то двумерный электростатический потенциал $\Phi$, создаваемый областью, является функционалом от ее формы. Потенциал $\Phi$ удовлетворяет уравнению Пуассона

$$
-\partial_{z} \partial_{\bar{z}} \Phi(z, \bar{z})= \begin{cases}\sigma(z, \bar{z}), & z=x+i y \in D_{+} \\ 0, & z=x+i y \in D_{-}\end{cases}
$$

и дается интегралом по области $D_{+}$:

$$
\Phi(z, \bar{z})=-\frac{2}{\pi} \int_{D_{+}} d^{2} z^{\prime} \sigma\left(z^{\prime}, \bar{z}^{\prime}\right) \ln \left|z-z^{\prime}\right| .
$$

В области $D_{-}$потенциал является гармонической функцией, которая представляется рядом

$$
\Phi^{-}(z, \bar{z})=-2 t_{0} \ln |z|+2 \operatorname{Re} \sum_{k>0} \frac{v_{k}}{k} z^{-k}
$$

при $z \rightarrow \infty$, где

$$
v_{k}=\frac{1}{\pi} \int_{D_{+}} z^{k} \sigma(z, \bar{z}) d^{2} z, \quad k>0,
$$

- моменты области $D_{+}$, вычисленные по отношению к плотности $\sigma$, и

$$
\pi t_{0}=\int_{D_{+}} \sigma(z, \bar{z}) d^{2} z
$$

- полньй заряд в $D_{+}$.

В области $D_{+}$потенциал $(11)$ представляется рядом $\Phi^{+}$, который имеет как гармонические, так и негармонические члены. Появление последних вызвано фоновым зарядом. Обозначим негармоническую часть через $U(z, \bar{z})$. Функция $U$ удовлетворяет уравнению Пуассона $\partial_{z} \partial_{\bar{z}} U(z, \bar{z})=\sigma(z, \bar{z})$ и фиксируется условием, что ее разложение вблизи нуля (напомним, что $0 \in D_{+}$) не содержит гармонических слагаемых. Например, если $\sigma(z, \bar{z})=1$, то $U(z, \bar{z})=z \bar{z}$. Мы примем, что $U$ имеет следующий общий вид:

$$
U(z, \bar{z})=-\sum_{m, n \geqslant 1} T_{m n} z^{m} \bar{z}^{n} .
$$

Разложение $\Phi$ вблизи $z=0$ получается из формулы (11) непосредственно. Результат имеет вид

$$
\Phi^{+}(z, \bar{z})=-U(z, \bar{z})-v_{0}+2 \operatorname{Re} \sum_{k>0} t_{k} z^{k}
$$


Негармонический вклад $U$ целиком обусловлен фоновым зарядом (см. выше) и не зависит от формы области. Наличие гранишы учитывается гармоническим вкладом. Коэффищиенты даются формулами

$$
t_{k}=\frac{1}{2 \pi i k} \oint_{\gamma} z^{-k} \partial_{z} U d z, \quad k>0
$$

Они дают дополнительный (к $\left.v_{k}\right)$ набор параметров, характеризуюших область $D_{+}$. Здесь и ниже мы используем те же обозначения для моментов $\left(t_{0}, t_{k}\right.$ и т.д.), что и в разделе 1 . В случае $\sigma=1$ они равны гармоническим моментам внешней области $D_{-}$. Наконец,

$$
v_{0}=\frac{2}{\pi} \int_{D_{+}} \ln |z| \sigma(z, \bar{z}) d^{2} z
$$

Заметим, что моменты $v_{k}(13)$ и $t_{0}(14)$ также допускают аналогичное представление в виде контурного интеграла:

$$
v_{k}=\frac{1}{2 \pi i} \oint_{\gamma} z^{k} \partial_{z} U d z, \quad k>0, \quad t_{0}=\frac{1}{2 \pi i} \oint_{\gamma} \partial_{z} U d z
$$

которое является простым следствием формулы Грина.

Так как потенциал и электрическое поле непрерывны на границе области, два дополнительных набора моментов связаны условиями

$$
\Phi^{+}=\Phi^{-}, \quad \partial_{z} \Phi^{+}=\partial_{z} \Phi^{-}, \quad z \in \gamma
$$

Обобшенная обратная задача теории потенциала состоит в том, чтобы найти кривую $\gamma$, если даны функция $\sigma$ и одна из функций $\Phi^{+}$или $\Phi^{-}$, т.е. один из бесконечных наборов моментов. В качестве независимых переменных выберем полный заряд $\pi t_{0}$ и коэффициенты $t_{k}, \bar{t}_{k} \quad(k \geqslant 1)$ мультипольного разложения $\Phi$ вблизи начала координат. При некоторых условиях, которые мы здесь не обсуждаем, эти переменные однозначно задают кривую, по крайней мере, если деформированная кривая, описываемая $t_{k}$, достаточно близка к некоторой фиксированной кривой. (Некоторые важные результаты о единственности решения обратной задачи теории потенциала можно найти в работах [12].) Стоит отметить одно из таких условий, характерное именно для случая неоднородного распределения фонового заряда. Для того чтобы задача была хорошо поставлена, необходимо потребовать, чтобы $\sigma(z, \bar{z}) \neq 0$ во всех точках кривой $\gamma$. В противном случае малая деформация кривой, локализованная вблизи нуля $\sigma$, не приведет к изменению потенциала $\Phi$, по крайней мере в первом порядке. Чтобы избежать этой трудности, можно потребовать, например, чтобы $\sigma(z, \bar{z})>0$.

Другими словами, при упомянутых выше условиях моменты $\left\{t_{k}\right\}_{k=0}^{\infty}$ являются хорошо определенными локальными координатами в пространстве аналитических кривых. Моменты $v_{j}$ тоже определяются ими, так что любой момент внутренности можно рассматривать как функцию от $t_{k}$. В этой работе мы для простоты предположим, что только конечное число коэффишиентов $t_{k}$ и $T_{m n}$ отлично от нуля. В этом случае ряд (16)- 
полином по $z, \bar{z}$, и, значит, он задает функцию $\Phi^{+}$для всех $z \in D_{+}$. Поскольку функция $\sigma$ вешественна, то $t_{0}, v_{0}$ - вешественные числа, а все остальные моменты, вообше говоря, комплексны.

Теперь перейдем к электростатическому вариационному принципу, упомянутому в начале этого раздела. Рассмотрим функционал энергии, описывающий заряд с плотностью $\rho(z, \bar{z})$ в фоновом потенциале $\Phi(11)$, который создается зарядом, распределенным в области $D_{+}$с плотностью $\sigma$ :

$$
\mathcal{E}(\rho)=-\frac{1}{\pi^{2}} \iint d^{2} z d^{2} z^{\prime} \rho(z, \bar{z}) \ln \left|z-z^{\prime}\right| \rho\left(z^{\prime}, \bar{z}^{\prime}\right)-\frac{1}{\pi} \int d^{2} z \rho(z, \bar{z}) \Phi(z, \bar{z})
$$

Первый член в (21) - это двумерная “кулоновская" энергия заряда, а второй - энергия взаимодействия с фоновым зарядом. Ясно, что минимум энергии достигается в случае, когда распределение заряда полностью нейтрализует фоновый заряд. Обозначим плотность этого распределения через $\rho_{0}: \rho_{0}=\sigma$ внутри области и $\rho_{0}=0$ вне ее, так что суммарная плотность заряда равна нулю. В минимуме функционал равен электростатической энергии $E$ фонового заряда с обратным знаком: $\min _{\rho} \mathcal{E}(\rho)=-E$, где

$$
E=-\frac{1}{\pi^{2}} \iint_{D_{+}} d^{2} z d^{2} z^{\prime} \sigma(z, \bar{z}) \ln \left|z-z^{\prime}\right| \sigma\left(z^{\prime}, \bar{z}^{\prime}\right)
$$

Проварьировав $\rho$ в формуле (21) и затем положив $\rho=\rho_{0}$, получим (16).

Найдем производные $E$ по $t_{k}$. Это можно сделать разными способами. Один из них заключается в прямом дифференцировании выражения (22). При этом нужно учесть вклад, связанный с малым изменением области при вариации $t_{k}$. Чтобы обойти эту проблему, мы используем тот же аргумент, что и в случае $\sigma=1$ (см. [3]). Именно, вместо прямого дифференцирования выражения (22) возьмем производную от функции $E$, представленную как экстремальное значение функционала $-\mathcal{E}(\rho)(21)$. При этом мы получим вклады двух типов: один происходит от явной зависимости $\Phi^{+}$от $t_{k}$, а другой от неявной зависимости $\rho_{0}$ от $t_{k}$ :

$$
\frac{\partial E}{\partial t_{k}}=-\left.\frac{\partial \mathcal{E}(\rho)}{\partial t_{k}}\right|_{\rho=\rho_{0}}=\frac{1}{\pi} \int_{D_{+}} d^{2} z \sigma(z, \bar{z}) \partial_{t_{k}} \Phi^{+}(z, \bar{z})-\left.\int d^{2} z \frac{\delta \mathcal{E}(\rho)}{\delta \rho(z, \bar{z})}\right|_{\rho=\rho_{0}} \frac{\delta \rho_{0}(z, \bar{z})}{\delta t_{k}}
$$

Поскольку мы рассматриваем минимум функционала $\mathcal{E}(\rho)$, то вариационная производная во втором слагаемом равна нулю, и этот член вклада не дает. Другими словами, искомая производная равна частной производной от $-\mathcal{E}$, вычисленной в экстремуме. Допустим, что нашими независимыми переменными являются $v_{0}$ и $t_{k}$, тогда частные производные от $\Phi^{+}$особенно просты: $\partial_{t_{k}} \Phi^{+}(z, \bar{z})=z^{k}, \partial_{v_{0}} \Phi^{+}(z, \bar{z})=1$. Подставляя их в интеграл, приходим к соотношениям

$$
\frac{\partial E}{\partial t_{k}}=v_{k}, \quad \frac{\partial E}{\partial \bar{t}_{k}}=\bar{v}_{k}, \quad \frac{\partial E}{\partial v_{0}}=-t_{0},
$$


где частные производные по $t_{k}$ берутся при фиксированных $v_{0}$ и $t_{j}(j \neq 0, k)$. Таким образом, дифференциал $d E$ имеет вид

$$
d E=\sum_{k>0}\left(v_{k} d t_{k}+\bar{v}_{k} d \bar{t}_{k}\right)-t_{0} d v_{0}
$$

Отметим, что при прямом вычислении производной от выражения (22) возникают вклады двух типов: один определяется взятием производной $\partial_{t_{k}} \Phi$, а другой связан с изменением формы области. Первый легко вычисляется и равен $v_{k} / 2$, значит, второй тоже равен $v_{k} / 2$. Однако этот результат не так легко получить прямым вычислением (пример такого вычисления содержится в приложении). Прямое доказательство формул для первых производных в случае $\sigma=1$ можно найти в работе [7].

Более естественно, однако, в качестве независимой переменной выбрать полный заряд, а не $v_{0}$, т.е. использовать вариационный принцип с фиксированным полным зарядом. Это достигается преобразованием Лежандра. Введем функцию $F=E+t_{0} v_{0}$. Ее дифференциал равен

$$
d F=\sum_{k>0}\left(v_{k} d t_{k}+\bar{v}_{k} d \bar{t}_{k}\right)+v_{0} d t_{0}
$$

а интегральное представление следует из (22):

$$
F=-\frac{1}{\pi^{2}} \iint_{D_{+}} d^{2} z d^{2} z^{\prime} \sigma(z, \bar{z}) \ln \left|z^{-1}-z^{\prime-1}\right| \sigma\left(z^{\prime}, \bar{z}^{\prime}\right) .
$$

Функция $F$ будет играть основную роль в дальнейшем. Это вещественнозначная функция моментов $t_{0}, t_{1}, t_{2}, \ldots$ Преобразовав соотношения (23) к новым переменным, получим основное свойство функции $F$ :

$$
\frac{\partial F}{\partial t_{k}}=v_{k}, \quad \frac{\partial F}{\partial \bar{t}_{k}}=\bar{v}_{k}, \quad \frac{\partial F}{\partial t_{0}}=v_{0},
$$

где производные по $t_{k}$ берутся при фиксированных $t_{j}(j \neq k)$. Само сушествование обшей потенциальной функции для моментов влечет соотношения симметрии для их производных, $\partial_{t_{n}} v_{k}=\partial_{t_{k}} v_{n}, \partial_{\bar{t}_{n}} v_{k}=\partial_{t_{k}} \bar{v}_{n}$, которые впервые были получены в работе [1] (в случае $\sigma=1$ ) другим способом.

Чтобы представить выражения (25) в более компактной форме, модифицируем потенциал $\Phi$ и введем функцию $\widetilde{\Phi}(z, \bar{z})=\Phi(z, \bar{z})+2 t_{0} \ln |z|+v_{0}$, так что

$$
\widetilde{\Phi}(z, \bar{z})=-\frac{2}{\pi} \int_{D_{+}} d^{2} \zeta \sigma(\zeta, \bar{\zeta}) \ln \left|z^{-1}-\zeta^{-1}\right|
$$

и

$$
F=\frac{1}{2 \pi} \int_{D_{+}} d^{2} z \sigma(z, \bar{z}) \widetilde{\Phi}^{+}(z, \bar{z}) .
$$


Эта модификация означает помешение в начало координат точечного заряда, нейтрализуюшего заряд области. Разложение модифицированного потенциала вблизи начала координат имеет вид

$$
\widetilde{\Phi}^{+}(z, \bar{z})=-U(z, \bar{z})+2 t_{0} \ln |z|+\sum_{k \geqslant 1}\left(t_{k} z^{k}+\bar{t}_{k} \bar{z}^{k}\right) .
$$

Здесь и ниже эта формула служит определением переменных $t_{k}$. Подобно $\Phi$, функция $\widetilde{\Phi}$ непрерывна на границе $\gamma=\partial D_{+}$вместе с первыми производными. При $z \in D_{-}$функция $\widetilde{\Phi}(z, \bar{z})$ гармонична. В терминах функции $\widetilde{\Phi}$ формулы $(25)$ могут быть записаны более компактно:

$$
\mathcal{D}(z, \bar{z}) F=\widetilde{\Phi}^{-}(z, \bar{z})
$$

где использован оператор $\mathcal{D}$, введенный в $(5)$, и предполагается, что $z$ лежит в области $D_{-}$. Обе части формулы надо понимать как ряды по $z$ и $\bar{z}$. В наших предположениях они сходятся в $D_{-}$.

В заключение этого раздела обсудим свойства однородности функции $F$. В предположении, что только конечное число величин $t_{k}$ отлично от нуля, можно подставить (16) в (24) и почленно проинтегрировать. Получим следующее соотношение:

$$
2 F=-\frac{1}{4 \pi} \int_{D_{+}} d^{2} z U \Delta U+t_{0} v_{0}+\sum_{k>0}\left(t_{k} v_{k}+\bar{t}_{k} \bar{v}_{k}\right),
$$

где $\Delta=4 \partial_{z} \partial_{\bar{z}}-$ оператор Лапласа. Вообще говоря, выражение (30) не имеет определенных свойств однородности, как функция от $t_{k}$. Тем не менее, если $\sigma$ однородна, то $F$ квазиоднородна. Например, если $\sigma(z, \bar{z})=|z|^{2 M-2}$ при целом $M$, то выражение (30) можно привести к виду

$$
4 M F=-t_{0}^{2}+2 M t_{0} \partial_{t_{0}} F+\sum_{k \geqslant 1}(2 M-k)\left(t_{k} \partial_{t_{k}} F+\bar{t}_{k} \partial_{\bar{t}_{k}} F\right),
$$

где учтены соотношения (25). Это равенство отражает свойства однородности моментов при растяжении $z \rightarrow \lambda z$ и означает, что $F$ - квазиоднородная функция степени $4 M$.

Более фундаментальное свойство однородности, справедливое для любых $\sigma$, можно получить, если обобщить определение функции $F$. Будем рассматривать параметры $T_{m n}$, которые задают $U$ или $\sigma$ (см. (15)) на равных правах с переменными $t_{k}$. Именно, отождествим $t_{k}=T_{k 0}, \bar{t}_{k}=T_{0 k}, t_{0}=T_{00}$ и рассмотрим $F$ как функцию всех $T_{m n}$, $m, n \geqslant 0$, которые теперь образуют расширенное множество независимых переменных. Те же вариационные аргументы, что и раньше, приводят к соотношениям

$$
\frac{\partial F}{\partial T_{m n}}=\frac{1}{\pi} \int_{D_{+}} z^{n} \bar{z}^{m} \sigma(z, \bar{z}) d^{2} z
$$

которые обобщают (25). Тогда (30) принимает вид настоящего условия однородности:

$$
2 F=\sum_{n, m \geqslant 0} T_{m n} \frac{\partial F}{\partial T_{m n}} .
$$


Тау-функцию можно ввести как $\tau=e^{F}$. Однако это обозначение введено здесь исключительно с целью подчеркнуть связь с уравнениями Хироты. В дальнейшем мы рассматриваем только функцию $F$, т.е. логарифм $\tau$-функции. Напомним, что сама $\tau$-функция не имеет хорошего бездисперсионного предела. В этом пределе имеет смысл только ее логарифм, умноженный на малый параметр дисперсии. Билинейные уравнения Хироты для $\tau$ переписываются как сильнонелинейные уравнения для $\ln \tau$. В следующем разделе мы выведем эти уравнения, исходя из определения функции $F$.

\section{3. ЗАДАЧА ДИРИХЛЕ И БЕЗДИСПЕРСИОННЫЕ УРАВНЕНИЯ ХИРОТЫ ДЛЯ ФУНКЦИИ $F$}

Бездисперсионные уравнения Хироты представляют собой соотношения между вторыми производными по $t_{k}$ от логарифма $\tau$-функции. Вторые производные от функции $F$ - это коэффициенты выражений вида $D\left(z_{1}\right) D\left(z_{2}\right) F$, где использован оператор $D(z)$, введенный в (4). Напомним, что первые производные $F$ нам уже известны $($ cм. $(29))$. Итак, мы должны найти $\mathcal{D}\left(z_{1}, \bar{z}_{1}\right) \widetilde{\Phi}\left(z_{2}, \bar{z}_{2}\right)$ для $z_{1}, z_{2} \in D_{-}$. Для этого используем следующее соображение общего характера.

Рассмотрим малую деформацию области $D_{+}$, при которой к исходной области добавляется бесконечно малый "нарост" ( "шишечка") произвольной формы площади $\epsilon$, локализованный вблизи точки $\xi \in \gamma$. Через $\delta_{\epsilon(\xi)}$ обозначим вариацию любой величины при такой деформации в первом порядке по $\epsilon$. Потенциал, создаваемый деформированной областью, равен $\widetilde{\Phi}+\delta_{\epsilon(\xi)} \widetilde{\Phi}$, где

$$
\delta_{\epsilon(\xi)} \widetilde{\Phi}(z, \bar{z})=-\frac{\epsilon}{\pi} \sigma(\xi, \bar{\xi}) \ln \left|z^{-1}-\xi^{-1}\right|^{2}
$$

- потенциал, создаваемьй “шишечкой”, что следует из явной формулы (26). Сравнив разложение $\delta_{\epsilon(\xi)} \widetilde{\Phi}(z, \bar{z})$ по $z$ при $z \rightarrow 0$ с выражением $(28)$, мы найдем вариации $t_{k}$ при деформации:

$$
\delta_{\epsilon(\xi)} t_{0}=\frac{\epsilon}{\pi} \sigma(\xi, \bar{\xi}), \quad \delta_{\epsilon(\xi)} t_{k}=\frac{\epsilon}{\pi k} \sigma(\xi, \bar{\xi}) \xi^{-k}, \quad k \geqslant 1
$$

Пусть $A$ - любая функция моментов, тогда ее вариация $\delta_{\epsilon(\xi)} A$ равна

$$
\delta_{\epsilon(\xi)} A=\partial_{t_{0}} A \delta_{\epsilon(\xi)} t_{0}+\sum_{k \geqslant 1}\left(\partial_{t_{k}} A \delta_{\epsilon(\xi)} t_{k}+\partial_{\bar{t}_{k}} A \delta_{\epsilon(\xi)} \bar{t}_{k}\right)
$$

что может быть записано в виде

$$
\delta_{\epsilon(\xi)} A=\frac{\epsilon}{\pi} \sigma(\xi, \bar{\xi}) \mathcal{D}(\xi, \bar{\xi}) A, \quad \xi \in \gamma
$$

Таким образом, мы видим, что для точки $\xi$, лежашей на гранище $\gamma=\partial D_{+}$, оператор $\mathcal{D}(\xi, \bar{\xi})$ имеет ясньй геометрический смысл: результат его действия на любую величину пропорционален вариации этой величины при добавлении "шишечки" в точке $\xi$. Другими словами, граничное значение функции $\mathcal{D}(z, \bar{z}) A$ равно $\pi \delta_{\epsilon(z)} A /(\epsilon \sigma(z, \bar{z})), z \in \gamma$. Для функций $A$ таких, что ряд $D(z) A$ сходится везде в $D$ - вплоть до границы, это замечание 
дает практический метод нахождения функции $\mathcal{D}(z, \bar{z}) A$. Именно, эта функция гармонична в $D_{-}$, а ее граничное значение дается формулой (33). Восстановление гармонической функции по ее граничному значению - это в точности предмет граничной задачи Дирихле. Этот метод оказывается особенно полезным в случае, когда левую часть (33) можно вычислить независимым образом.

В качестве простейшего примера легко найдем $\delta_{\epsilon(\xi)} F=(\epsilon / \pi) \sigma(\xi, \bar{\xi}) \widetilde{\Phi}(\xi, \bar{\xi})$, что сразу следует из интегрального представления $\widetilde{\Phi}$. В этом случае гармоническое продолжение не требует никаких дополнительных действий, так как сама функция $\widetilde{\Phi}$ гармонична в $D_{-}$. Таким образом, мы воспроизвели результат (29).

Перейдем ко вторым производным. Как следует из (33), граничное значение функции $\mathcal{D}(\xi, \bar{\xi}) \mathcal{D}(z, \bar{z}) F=\mathcal{D}(\xi, \bar{\xi}) \widetilde{\Phi}(z, \bar{z})$, которую здесь следует рассматривать как функцию от $\xi$, дается уравнением (32) при $z$, лежашем в области $D_{-}$:

$$
\mathcal{D}(\xi, \bar{\xi}) \mathcal{D}(z, \bar{z}) F=-2 \ln \left|z^{-1}-\xi^{-1}\right|, \quad \xi \in \gamma
$$

Мы здесь не обсуждаем сходимость ряда $D(\xi) \widetilde{\Phi}$ в $D_{-}$, которую необходимо предположить, чтобы иметь возможность применения правила гармонического продолжения, объясненного вьше. Чтобы быть уверенными, что класс областей, обладающих этим свойством, не пуст, можно иметь в виду области и распределения фонового заряда, достаточно близкие к равномерно заряженному диску. В этом случае доказать сходимость можно с помощью некоторых стандартных оценок. Правая часть (34) не гармонична в $D_{-}$в том виде, как она написана, так как имеет логарифмическую особенность при $\xi=z$. Чтобы найти гармоническое продолжение этой функции, добавим к (34) функцию, гармоническую везде в $D_{-}$, кроме точки $z$, где она имеет логарифмическую особенность вида $+2 \ln \left|z^{-1}-\xi^{-1}\right|$ (которая, таким образом, сокращает особенность в (34)), и равную нулю на границе. По определению функции Грина задачи Дирихле (см. раздел 1$)$ такая функция есть функция Грина $G(z, \xi)(2)$. Отсюда мы получаем формулу (7). Устремив $z_{2}$ к бесконечности, а затем отделив голоморфную часть по $z_{1}$, приходим к выражению (6) для конформного отображения. Выделив голоморфную и антиголоморфную части (7) по обеим переменным и воспользовавшись формулой (2), получим

$$
\begin{gathered}
\ln \frac{w\left(z_{1}\right)-w\left(z_{2}\right)}{z_{1}-z_{2}}=-\frac{1}{2} \partial_{t_{0}}^{2} F+D\left(z_{1}\right) D\left(z_{2}\right) F \\
\ln \left(1-\frac{1}{w\left(z_{1}\right) \overline{w\left(z_{2}\right)}}\right)=-D\left(z_{1}\right) D\left(z_{2}\right) F .
\end{gathered}
$$

Перейдя к пределу при $z_{2} \rightarrow \infty$ в соотношении (35), получим другую полезную формулу для конформного отображения:

$$
w(z)=e^{-\frac{1}{2} \partial_{t_{0}}^{2} F}\left(z-\left(\partial_{t_{0}}+D(z)\right) \partial_{t_{1}} F\right)
$$

Все эти формулы имеют одинаковый вид для любой функции $\sigma$.

Теперь уже нетрудно увидеть, как возникают уравнения Хироты. Они получаются путем подстановки формулы (6) в равенства типа (35), (36), что приводит к исключению 
из них $w(z)$. Так получаются уравнения (8), (9), которые и являются бездисперсионным пределом билинейных уравнений Хироты для иерархии уравнений двумеризованной цепочки Тоды. Для вывода другого уравнения Хироты даже не требуется использования формулы (6): напишем уравнения (35) для каждой пары из точек $z_{1}, z_{2}, z_{3}$, возьмем экспоненту от обеих частей каждого равенства и сложим их. Получим уравнение

$$
\left(z_{1}-z_{2}\right) e^{D\left(z_{1}\right) D\left(z_{2}\right) F}+\left(z_{2}-z_{3}\right) e^{D\left(z_{2}\right) D\left(z_{3}\right) F}+\left(z_{3}-z_{1}\right) e^{D\left(z_{3}\right) D\left(z_{1}\right) F}=0 .
$$

Это бездисперсионная иерархия Кадомцева-Петвиашвили (голоморфный по отношению к переменным $t_{k}$ сектор цепочки Тоды), записанная в наиболее симметричной форме. В литературе [13], [11] известна несколько иная версия, которая получается отсюда при $z_{3} \rightarrow \infty$.

Три уравнения (8), (9) и (38) (вместе с их антиголоморфными версиями) должны пониматься как бесконечный набор соотношений на вторые производные от $F$. Взятые все вместе, они образуют интегрируемую иерархию. Эти соотношения получаются разложением обеих частей в ряды по $z_{i}$ и сравнением коэффициентов. Например, сравнение лидируюших членов в обеих частях уравнения (9) при $z_{1}, z_{2} \rightarrow \infty$ дает первое уравнение иерархии. Оно представляет собой бездисперсионный предел уравнения Тоды: $\partial_{t_{1} \bar{t}_{1}}^{2} F=e^{\partial_{t_{0}}^{2} F} .\left(\right.$ Если ввести поле $\phi=v_{0}=\partial_{t_{0}} F$, имеем $\partial_{t_{1} \bar{t}_{1}}^{2} \phi=\partial_{t_{0}} e^{\partial_{t_{0}} \phi}$.) Следуюшие за лидируюшими члены дают уравнение $\partial_{t_{2} \bar{t}_{1}}^{2} F=2 \partial_{t_{0} t_{1}}^{2} F \partial_{t_{1} \bar{t}_{1}}^{2} F$.

Сделаем важное замечание. Все формулы для вторых производных от $F$, полученные в этом разделе, имеют один и тот же вид для любой функции $\sigma$, т.е. $\sigma$ не входит в них явно. В самом деле, рассмотрим, например, соотношения (35), (36). Их левые части, коль скоро они выражены через конформное отображение, определяются только формой области. При фиксированной форме области они одинаковы для любой плотности фонового заряда. Ясно, что это утверждение остается в силе и применительно к производяшей формуле для вторых производных $(7)$. Заметим, что сама функция $F$ зависит от выбора $\sigma$.

Это замечание можно сформулировать как некоторое свойство ковариантности вторых производных. Пусть $\widehat{F}, \hat{t}_{k}$ - зависимые и независимые переменные, относящиеся к другой функции плотности, равной $\hat{\sigma}(z, \bar{z})$. Как следует из предыдушего замечания, вторые производные $F$ ковариантны в следующем смысле:

$$
\frac{\partial^{2} \widehat{F}}{\partial \hat{t}_{j} \partial \hat{t}_{k}}=\frac{\partial^{2} F}{\partial t_{j} \partial t_{k}}
$$

при условии, что обе части вычислены для одной и той же области $D_{+} \cdot$ Справедлива также аналогичная формула для смешанных производных по $t_{j}, \bar{t}_{k}$. Подчеркнем, что это свойство имеет место только для вторых производных. Например, как видно из (29), производная $\partial_{\hat{t}_{j}} \widehat{F}$, вообше говоря, не равна $\partial_{t_{j}} F$, поскольку потенциал $\widetilde{\Phi}^{-}$зависит не только от самой области, но и от $\sigma$.

Уравнения Хироты инвариантны по отношению к изменению плотности $\sigma$. В то же время любое частное решение, описываюшее конформные отображения и задачу Дирихле, определяется фоновым зарядом. Мы приходим к выводу, что функция $\sigma$ параметризует различные решения уравнений Хироты. В разделе 5 мы вернемся к этому 
вопросу с другой точки зрения. Если понимать $F$ как функцию всех $T_{m n}$ (см. конец раздела 2), она дает решение расширенной иерархии Тоды, введенной в работе [14] в контексте двухматричной модели.

Сделаем краткое замечание по поводу билинейных уравнений Хироты и их бездисперсионного предела. Пусть $\hbar$ - некоторый параметр. Уравнения Хироты записываются для $\tau$-функции $\tau_{\hbar}$, которая зависит от времен $t_{k}$ и от параметра $\hbar$. Мы приводим уравнения в том же порядке, что и в бездисперсионном случае, и пользуемся теми же обозначениями. Уравнения (8), (9) заменяются на соотношения

$$
\begin{aligned}
& z_{1}\left(e^{\hbar\left(\partial_{t_{0}}-D\left(z_{1}\right)\right)} \tau_{\hbar}\right)\left(e^{-\hbar D\left(z_{2}\right)} \tau_{\hbar}\right)-z_{2}\left(e^{\hbar\left(\partial_{t_{0}}-D\left(z_{2}\right)\right)} \tau_{\hbar}\right)\left(e^{-\hbar D\left(z_{1}\right)} \tau_{\hbar}\right)= \\
& \quad=\left(z_{1}-z_{2}\right)\left(e^{-\hbar\left(D\left(z_{1}\right)+D\left(z_{2}\right)\right)} \tau_{\hbar}\right)\left(e^{\hbar \partial_{t_{0}}} \tau_{\hbar}\right), \\
& \left(e^{-\hbar D\left(z_{1}\right)} \tau_{\hbar}\right)\left(e^{-\hbar D\left(\bar{z}_{2}\right)} \tau_{\hbar}\right)-\tau_{\hbar}\left(e^{\hbar\left(\bar{D}\left(\bar{z}_{2}\right)-D\left(z_{1}\right)\right)} \tau_{\hbar}\right)= \\
& =\left(z_{1} \bar{z}_{2}\right)^{-1}\left(e^{-\hbar\left(\partial_{t_{0}}+D\left(z_{1}\right)\right)} \tau_{\hbar}\right)\left(e^{\hbar\left(\partial_{t_{0}}+\bar{D}\left(\bar{z}_{2}\right)\right)} \tau_{\hbar}\right),
\end{aligned}
$$

соответственно. Дисперсионный аналог уравнения (38) имеет вид

$$
\left(z_{1}-z_{2}\right)\left(e^{-\hbar\left(D\left(z_{1}\right)+D\left(z_{2}\right)\right)} \tau_{\hbar}\right)\left(e^{-\hbar D\left(z_{3}\right)} \tau_{\hbar}\right)+\text { цикл }\left(z_{1}, z_{2}, z_{3}\right)=0 .
$$

Здесь $e^{\hbar D(z)}$ - операторы сдвига, так что эти уравнения являются разностными. Для более наглядной записи введем векторные поля $\partial_{x_{i}}=-D\left(z_{i}\right)$. В переменных $x_{i}$ уравнения выглядят более знакомо. Например, уравнение (42) приобретает вид

$$
\left(z_{1}-z_{2}\right) \tau_{\hbar}\left(x_{1}+\hbar, x_{2}+\hbar, x_{3}\right) \tau_{\hbar}\left(x_{1}, x_{2}, x_{3}+\hbar\right)+\text { цикл }(1,2,3)=0 .
$$

Эти билинейные разностные уравнения для $\tau$-функции обладают многими замечательными свойствами. Уравнение (42) впервые появилось в статье Хироты [15]. Более подробную информацию о разностных уравнениях Хироты можно найти, например, в обзоpe $[16]$.

Из вышеприведенных формул ясно, что параметр $\hbar$ играет роль постоянной решетки. В бездисперсионном пределе разностные уравнения становятся дифференциальными. Этот предел хорошо определен на классе решений таких, что сушествует конечный предел

$$
F=\lim _{\hbar \rightarrow 0} \hbar^{2} \ln \tau_{\hbar}
$$

Переписав уравнения Хироты как уравнения на $\ln \tau_{\hbar}$ и выделив лидирующие вклады при $\hbar \rightarrow 0$, придем к бездисперсионным уравнениям Хироты для $F$. Различные аспекты математической теории бездисперсионных интегрируемых уравнений обсуждались в работах [17], [18], [11], [13]. 


\section{4. ИНТЕГРИРУЕМАЯ СТРУКТУРА КОНФОРМНЫХ ОТОБРАЖЕНИЙ В ФОРМАЛИЗМЕ ТАКАСАКИ-ТАКЕБЕ}

Подход предыдушего раздела дает, возможно, самый простой способ увидеть связь меж ду задачей Дирихле и уравнениями Хироты. Однако такие более привычные атрибуты интегрируемых моделей, как, скажем, представление Лакса, остаются при этом за рамками рассмотрения. В этом разделе мы обсудим альтернативный подход, который позволяет выявить интегрируемую структуру конформных отображений с помощью представления типа Лакса для обратного отображения, которое мы обозначим $z(w)$. В нашей нормировке имеем следуюший общий вид обратного отображения:

$$
z(w)=r w+\sum_{j=0}^{\infty} u_{j} w^{-j},
$$

где $r$ - вешественное положительное число. При значении $w$, лежащем на единичной окружности, соотношение (45) дает параметризацию кривой.

Введем еще один новый объект. Пусть $S(z)$ - аналитическое продолжение функции $\partial_{z} U$ с кривой $\gamma$ :

$$
S(z)=\partial_{z} U(z, \bar{z}), \quad z \in \gamma .
$$

Можно доказать, что для аналитических кривых функция $S(z)$ голоморфна в некоторой трубчатой окрестности кривой $\gamma$. Эта функция оказывается очень полезной с технической точки зрения. Мы будем называть ее обобщенной функиией Швариа, или для краткости просто функцией Шварца (последнее название обычно используется в случае $\sigma=1$, см., например, книгу [19]). Сопоставив это определение с определением моментов через контурные интегралы (17), (19), получим разложение функции Шварца в ряд Лорана:

$$
S(z)=\sum_{k=1}^{\infty} k t_{k} z^{k-1}+\frac{t_{0}}{z}+\sum_{k=1}^{\infty} v_{k} z^{-k-1} .
$$

Мы видим, что $S(z)$ является производящей функцией всех моментов $t_{k}, v_{k}$. Будем считать ее функцией моментов $t_{k}: S(z)=S\left(t_{k}, z\right)$. Если даны коэффициенты $T_{m n}(15)$ и функция Шварца, то кривая $\gamma$ определяется уравнением (46).

Следуя работе [11], мы введем бездисперсионную иерархию уравнений двумеризованной цепочки Тоды, взяв в качестве исходных следуюшие данные. Пусть даны четыре ряда Лорана вида

$$
\begin{array}{ll}
L=r w+\sum_{j=0}^{\infty} u_{j} w^{-j}, & M=\sum_{k=1}^{\infty} k t_{k} L^{k}+t_{0}+\sum_{k=1}^{\infty} v_{k} L^{-k}, \\
\bar{L}=r w^{-1}+\sum_{j=0}^{\infty} \bar{u}_{j} w^{j}, & \bar{M}=\sum_{k=1}^{\infty} k \bar{t}_{k} \bar{L}^{k}+t_{0}+\sum_{k=1}^{\infty} \bar{v}_{k} \bar{L}^{-k} .
\end{array}
$$

Здесь коэффициенты $t_{0}, t_{k}, \bar{t}_{k}-$ независимые переменные, а все остальные коэффициенты $\left(r, u_{j}, \bar{u}_{j}, v_{j}, \bar{v}_{j}\right)-$ зависимые переменные. Наложив на эти ряды Лорана некоторые условия, мы собираемся изучать, как последние зависят от первых. 
Напомним теорему Такасаки-Такебе [11]. Введем скобку Пуассона

$$
\{f, g\}=w \frac{\partial f}{\partial w} \frac{\partial g}{\partial t_{0}}-w \frac{\partial g}{\partial w} \frac{\partial f}{\partial t_{0}} .
$$

Пусть $f, g$ - каноническая пара, т.е. $\left\{f\left(w, t_{0}\right), g\left(w, t_{0}\right)\right\}=f\left(w, t_{0}\right)$. Предположим, что четыре ряда Лорана $L, \bar{L}, M, \bar{M}$, введенные выше, связаны функциональными соотношениями

$$
\bar{L}=f^{-1}(L, M), \quad \bar{M}=g(L, M) .
$$

Тогда справедливы следуюшие утверждения:

А. Пары $L, M$ и $(\bar{L})^{-1}, \bar{M}$ канонические: $\{L, M\}=L,\left\{\bar{L}^{-1}, \bar{M}\right\}=\bar{L}^{-1}$.

Б. Каждый из четырех рядов Лорана удовлетворяет уравнениям Лакса-Сато

$$
\frac{\partial X}{\partial t_{n}}=\left\{H_{n}, X\right\}, \quad \frac{\partial X}{\partial \bar{t}_{n}}=-\left\{\bar{H}_{n}, X\right\},
$$

где $X$ означает любой из рядов $L, \bar{L}, M, \bar{M}$ и

$$
H_{n}=\left(L^{n}\right)_{\geqslant 1}+\frac{1}{2}\left(L^{n}\right)_{0}, \quad \bar{H}_{n}=\left(\bar{L}^{n}\right)_{\leqslant-1}+\frac{1}{2}\left(\bar{L}^{n}\right)_{0}, \quad n \geqslant 1,
$$

где $(\ldots) \geqslant 1 \quad((\ldots) \leqslant-1)$ означает часть ряда Лорана, содержащую строго положительные (строго отрицательные) степени $w$, а постоянный член ряда обозначен через $(\ldots)_{0}$.

В. Сушествует функция $F$ переменных $t_{k}, \bar{t}_{k}$ и $t_{0}$ такая, что $v_{k}=\partial_{t_{k}} F, \bar{v}_{k}=\partial_{\bar{t}_{k}} F$, и функция $F$ удовлетворяет бездисперсионным уравнениям Хироты.

Эта теорема доказана в работе [11]. Доказательство утверждения А заключается в дифференцировании соотношений (51) по $w, t_{0}$ и сравнении результатов. Взяв производные от (51) по $t_{n}, \bar{t}_{n}$ и воспользовавшись утверждением А, получим утверждение Б. Для доказательства утверждения В нужно вычислить производную $\partial M / \partial t_{k}$ при постоянном $w$. Используя уравнения Лакса-Сато (52), получим $\partial_{t_{j}} v_{k}=\left(w L^{k} d H_{j}\right)_{0}$. Далее, из этого представления и соотношений (53) следует симметрия $\partial_{t_{j}} v_{k}=\partial_{t_{k}} v_{j}$, которая, в свою очередь, означает существование обшей потенциальной функции для моментов $v_{k}$. Доказательство уравнений Хироты для функции $F$, которое в принципе можно найти в работе [11], слишком неявное. Более прямые аргументы приведены в статье [13] (см. также [3]).

Из всего вьшеизложенного следует, что $L$ и $\bar{L}$ - две функции Лакса бездисперсионной иерархии Тоды. Уравнения иерархии записываются для “потеншиалов" $r,\left\{u_{n}\right\},\left\{\bar{u}_{n}\right\}$ как функций времен $t_{0},\left\{t_{n}\right\},\left\{\bar{t}_{n}\right\}$. Эти времена явно входят в ряды Лорана $M, \bar{M}$, которые представляют собой функции Орлова-Шульмана [20] для этой иерархии. Теорема Такасаки-Такебе утверждает, что различные решения иерархии находятся в однозначном соответствии с каноническими парами функций $f, g$.

Замечательным образом этот формализм оказался очень хорошо приспособленным для описания интегрируемой структуры конформных отображений. Отождествление 
объектов из теории конформных отображений с введенными выше рядами Лорана осушествляется следуюшим образом:

$$
L(w)=z(w), \quad \bar{L}(w)=\bar{z}\left(w^{-1}\right), \quad M(L)=z S(z), \quad \bar{M}(\bar{L})=\bar{z} \bar{S}(\bar{z})
$$

(для ряда $f(z)=\sum f_{j} z^{j}$ мы пишем $\left.\bar{f}(z)=\sum \bar{f}_{j} z^{j}\right)$. С учетом формулы $S(z)=\partial_{z} U(z, \bar{z})$ это означает, что мы имеем следующие функциональные соотношения между $L, \bar{L}$, $M, \bar{M}$ :

$$
M=L \frac{\partial U(L, \bar{L})}{\partial L}, \quad \bar{M}=\bar{L} \frac{\partial U(L, \bar{L})}{\partial \bar{L}} .
$$

Заметим, что соотношения (54) дают эквивалентное представление канонической пары из теоремы Такасаки-Такебе. Именно, формулы (54) указывают на то, что $U(L, \bar{L})$ есть производящая функция канонического преобразования $L, M \rightarrow \bar{L}^{-1}, \bar{M}$, и, следовательно, неявно задают соответствуюшую каноническую $(f, g)$-пару. Действительно, положим $M=F(L, \bar{L}), \bar{M}=G(L, \bar{L})$ и найдем скобку Пуассона между $\bar{L}^{-1}$ и $\bar{M}$. Для краткости будем писать $\bar{L}=\varphi(L, M)$, где $\varphi$ - функция, неявно заданная соотношением $M=F(L, \bar{L})$. Имеем

$$
\frac{\partial \bar{L}}{\partial L} \frac{\partial \bar{M}}{\partial M}-\frac{\partial \bar{L}}{\partial M} \frac{\partial \bar{M}}{\partial L}=\frac{\partial \varphi}{\partial L} \frac{\partial G}{\partial \bar{L}} \frac{\partial \varphi}{\partial M}-\frac{\partial \varphi}{\partial M}\left(\frac{\partial G}{\partial \bar{L}}+\frac{\partial G}{\partial \bar{L}} \frac{\partial \varphi}{\partial L}\right)=-\frac{\partial \varphi}{\partial M} \frac{\partial G}{\partial \bar{L}} .
$$

Следовательно,

$$
\left\{\bar{L}^{-1}, \bar{M}\right\}=L \bar{L}^{-2} \frac{\partial \varphi}{\partial M} \frac{\partial G}{\partial \bar{L}}=\frac{L}{\bar{L}^{2}} \frac{\partial G / \partial L}{\partial F / \partial \bar{L}},
$$

где последнее равенство следует из формулы

$$
\frac{\partial \varphi}{\partial M}=\left(\frac{\partial \bar{L}}{\partial M}\right)_{L=\text { const }}=\left(\frac{\partial F}{\partial \bar{L}}\right)^{-1} .
$$

Далее, взяв $F, G$ из (54), получим $\left\{\bar{L}^{-1}, \bar{M}\right\}=\bar{L}^{-1}$, т.е. преобразование (54) в самом деле каноническое.

Скобка Пуассона $\left\{L, \partial_{L} U(L, \bar{L})\right\}=1$, что следует из $\{L, M\}=L$. Это можно переписать как

$$
\{L, \bar{L}\}=\frac{1}{\sigma(L, \bar{L})},
$$

что есть одна из форм так называемого струнного уравнения.

\section{5. ЗАКЛЮЧЕНИЕ}

Мы показали, что некоторые классические задачи комплексного анализа обладают интегрируемой структурой, которая совпадает с интегрируемой структурой солитонных уравнений в бездисперсионном пределе. По данной односвязной области в комплексной плоскости и вспомогательной вешественно-аналитической функции $\sigma(z, \bar{z})$, заданной на всей комплексной плоскости, мы построили решение иерархии уравнений бездисперсионной двумеризованной цепочки Тоды. Независимыми потоками иерархии являются моменты области, или коэффициенты мультипольного разложения, определенные 
по отношению к функции $\sigma$, которая в контексте теории потенциала играет роль плотности фонового заряда. Зависимые переменные - это набор коэффициентов мультипольного разложения, дополнительный к исходному. Энергия фонового заряда как функция моментов области есть логарифм $\tau$-функции иерархии. Эта функция удовлетворяет бездисперсионным уравнениям Хироты. Ее производные по моментам дают формальные решения задачи о конформном отображении, граничной задачи Дирихле и двумерной обратной задачи теории потенциала. Интересный вопрос, к которому мы надеемся вернуться в последующих публикациях, - это обратная задача, связанная с обозначенной выше схемой: начав с какого-нибудь решения бездисперсионных уравнений Хироты, связать с ним некоторую граничную задачу или задачу о конформном отображении и восстановить функцию $\sigma$. Такая интерпретация может быть полезна для лучшего понимания бездисперсионных интегрируемых иерархий.

Благодарности. Автор благодарен организаторам конференции "Классические и квантовые интегрируемые системы" в Протвино за возможность представить эти результаты, а также П. Вигману, И. Кричеверу, А. Маршакову и М. Минееву-Вайнштейну за полезные обсуждения. Работа была частично поддержана грантами CRDF № RP1-2102, INTAS-99-0590 и РФФИ № 00-02-16477.

ПРИЛОЖЕНИЕ

Приведем прямое доказательство формул (25) для первых производных функции $F$ по $t_{k}$. Мы используем обозначения раздела 2.

Рассмотрим вариацию $\delta_{k} F(k>0)$ функции

$$
F=\frac{1}{2 \pi} \int_{D_{+}} \widetilde{\Phi}(z, \bar{z}) \sigma(z, \bar{z}) d^{2} z
$$

при малом изменении $t_{k} \rightarrow t_{k}+\delta t_{k}: F \rightarrow F+\delta_{k} F$. Все остальные моменты $t_{j}, j \neq k$, $j \geqslant 0$, остаются постоянными. Имеем

$$
\delta_{k} F=\frac{1}{2 \pi} \int_{D_{+}} \delta_{k} \widetilde{\Phi}^{+}(z, \bar{z}) \sigma(z, \bar{z}) d^{2} z+\frac{1}{2 \pi} \int_{\delta_{k} D_{+}} \widetilde{\Phi}(z, \bar{z}) \sigma(z, \bar{z}) d^{2} z,
$$

где $\delta_{k} D_{+}-$вариация области при изменении $t_{k}$ (более точно, $\int_{\delta_{k} D_{+}}$означает здесь разность $\left.\int_{D_{+}\left(t_{k}+\delta t_{k}\right)}-\int_{D_{+}\left(t_{k}\right)}\right)$. Обозначим через $I_{1}$ и $I_{2}$ первый и второй члены этой формулы. Первый член $I_{1}$ легко вычисляется. Поскольку $\delta_{k} \widetilde{\Phi}^{+}(z, \bar{z})=z^{k} \delta t_{k}+\bar{z}^{k} \delta \bar{t}_{k}$, то первый член равен

$$
I_{1}=\frac{1}{2 \pi} \int_{D_{+}} \delta_{k} \widetilde{\Phi}^{+}(z, \bar{z}) \sigma(z, \bar{z}) d^{2} z=\frac{1}{2}\left(v_{k} \delta t_{k}+\bar{v}_{k} \delta \bar{t}_{k}\right) .
$$

Перейдем теперь к члену $I_{2}$. Поскольку интеграл берется по малой окрестности кривой $\gamma=\partial D_{+}$, то можно заменить потенциал $\widetilde{\Phi}$ на $\widetilde{\Phi}^{-}$, который в силу условий $(20)$ совпадает в этой окрестности с $\widetilde{\Phi}^{+}$с точностью до членов второго порядка. Напомним, что $\widetilde{\Phi}^{-}(z, \bar{z})=v_{0}+2 \operatorname{Re} \omega(z)$, где функция

$$
\omega(z)=\sum_{k \geqslant 1} \frac{v_{k}}{k} z^{-k}
$$


голоморфна в $D_{-}$и регулярна на бесконечности. В наших предположениях все особенности функции $\omega$ лежат в некоторой области $B \subset D_{+}$такой, что $R=D_{+} \backslash B$ - трубчатая окрестность кривой. Другими словами, функция $\omega(z)$ может быть аналитически продолжена через кривую $\gamma$ внутрь области $R$. Учтем также, что мы работаем в пространстве аналитических кривых. Можно доказать, что для кривых, принадлежаших малой, но конечной окрестности точки этого пространства, соответствующей данной кривой, область $B$ можно выбрать не зависяшей от $t_{k}$.

В первом порядке по $\delta t_{k}$ можно записать

$$
I_{2}=\frac{v_{0}}{2 \pi} \int_{\delta_{k} D_{+}} \sigma(z, \bar{z}) d^{2} z+\frac{1}{\pi} \operatorname{Re} \int_{\delta_{k} R} \omega(z) \sigma(z, \bar{z}) d^{2} z .
$$

Первый интеграл в правой части есть вариация $t_{0}$, и, следовательно, он равен нулю, если $t_{0}$ остается постоянным. Таким образом, получаем

$$
\begin{aligned}
I_{2} & =\frac{1}{\pi} \operatorname{Re} \int_{D_{+}\left(t_{k}+\delta t_{k}\right) \backslash B} \omega(z) \sigma(z, \bar{z}) d^{2} z-\frac{1}{\pi} \operatorname{Re} \int_{D_{+}\left(t_{k}\right) \backslash B} \omega(z) \sigma(z, \bar{z}) d^{2} z= \\
& =\operatorname{Re} \oint_{\partial R\left(t_{k}+\delta t_{k}\right)} \frac{d z}{2 \pi i} \omega(z) \partial_{z} U(z, \bar{z})-\operatorname{Re} \oint_{\partial R\left(t_{k}\right)} \frac{d z}{2 \pi i} \omega(z) \partial_{z} U(z, \bar{z}) .
\end{aligned}
$$

Второе равенство следует из формулы Стокса. Граница области $R$ состоит из двух кривых, внутренней и внешней. Внутренняя граница одинакова в обоих интегралах, и ее вклад сокрашается. Внешней гранищей является кривая $\gamma\left(t_{k}+\delta t_{k}\right)$ в первом интеграле и кривая $\gamma\left(t_{k}\right)$ во втором. Следовательно, используя определение обобшенной функции Шварца (46), имеем

$$
I_{2}=\operatorname{Re} \oint_{\gamma\left(t_{k}+\delta t_{k}\right)} \frac{d z}{2 \pi i} \omega(z) S\left(t_{k}+\delta t_{k}, z\right)-\operatorname{Re} \oint_{\gamma\left(t_{k}\right)} \frac{d z}{2 \pi i} \omega(z) S\left(t_{k}, z\right)
$$

Поскольку функция $S(z)$ голоморфна в области $R$, контур интегрирования можно выбрать совпадаюшим с $\gamma=\gamma\left(t_{k}\right)$ в обоих интегралах. Отсюда находим, что в силу (47)

$$
I_{2}=\operatorname{Re}\left(\delta t_{k} \oint_{\gamma} \frac{d z}{2 \pi i} \omega(z) \frac{\partial S(z)}{\partial t_{k}}\right)=\frac{1}{2}\left(v_{k} \delta t_{k}+\bar{v}_{k} \delta \bar{t}_{k}\right) .
$$

Сложив вклады $I_{1}$ и $I_{2}$, получим $\delta_{k} F=v_{k} \delta t_{k}+\bar{v}_{k} \delta \bar{t}_{k}$, что представляет собой искомый результат для $k \geqslant 1$. Вычисление производной по $t_{0}$ проводится аналогично. 


\section{Список литературы}

[1] M. Mineev-Weinstein, P. B. Wiegmann, A. Zabrodin. Phys. Rev. Lett. 2000. V. 84. P. 5106-5109.

[2] P. B. Wiegmann, A. Zabrodin. Commun. Math. Phys. 2000. V. 213. P. 523-538.

[3] I. Kostov, I. Krichever, M. Mineev-Weinstein, P. B. Wiegmann, A. Zabrodin. $\tau$-function for analytic curves. hep-th/0005259.

[4] А. Гурвии, Р. Курант. Теория функций. М.: Наука, 1968.

[5] E. Hille. Analytic Function Theory. V.II. Boston: Ginn and Company, 1962.

[6] L. Zalcman. Contemp. Math. 1987. V. 63. P. 337-349.

[7] L. Takhtajan. Free bosons and tau-functions for compact Riemann surfaces and closed smooth Jordan curves. I. Current correlation functions. math.QA/0102164.

[8] И. М. Кричевер. Функц. анализ и его прилож. 1988. Т. 22. № 3. С. 37-52; I. Krichever. Commun. Pure Appl. Math. 1992. V. 47. P. 437-476.

[9] Б. А. Дубровин, С. П. Новиков. ДАН СССР. 1983. Т. 270. С. 781-785.

[10] С. П. Царев. ДАН СССР. 1985. Т. 282. С. 534-537.

[11] K. Takasaki, T. Takebe. Rev. Math. Phys. 1995. V. 7. P. 743-808.

[12] П. С. Новиков. ДАН СССР. 1938. Т. 18. № 3. С. 165-168; M. Sakai. Proc. Am. Math. Soc. 1978. V. 70. P. 35-38; V. Strakhov, M. Brodsky. SIAM J. Appl. Math. 1986. V. 46. P. 324-344.

[13] R. Carroll, Y. Kodama. J. Phys. A. 1995. V. 28. P. 6373-6388.

[14] L. Bonora, C.S. Xiong. Nucl. Phys. B. 1995. V. 344. P. 408-444.

[15] R. Hirota. J. Phys. Soc. Japan. 1981. V. 50. P. 3785-3791.

[16] A. В. Забродин. ТМФ. 1997. Т. 113. С. 179-230.

[17] J. Gibbons, Y. Kodama. Phys. Lett. A. 1989. V. 135. P. 167-170; Solving dispersionless Lax equations. In: Singular Limits of Dispersive Waves. Proc. of NATO ASI. Ed. N. Ercolani. New York: Plenum, 1994. P. 61-66.

[18] B. A. Dubrovin. Commun. Math. Phys. 1992. V. 145. P. 195-207; Geometry of 2D topological field theories. In: Integrable Systems and Quantum Groups (Montecatini Terme, 1993). Lect. Notes Math. V. 1620. Eds. M. Francaviglia at al. Berlin: Springer, 1996. P. 120-348; hep-th/9407018.

[19] P. J. Davis. The Schwarz Function and its Applications. The Carus Mathematical Monographs. № 17. Washington, DC: The Math. Association of America, 1974.

[20] A. Orlov, E. Shulman. Lett. Math. Phys. 1986. V. 12. P. 171-179. 